\title{
Zeta Function Zeros, Powers of Primes, and Quantum Chaos
}

\author{
Jamal Sakhr, Rajat K. Bhaduri, and Brandon P. van Zyl \\ Department of Physics and Astronomy, \\ McMaster University, Hamilton, Ontario, Canada L8S $4 M 1$
}

(Dated: November 20, 2018)

\begin{abstract}
We present a numerical study of Riemann's formula for the oscillating part of the density of the primes and their powers. The formula is comprised of an infinite series of oscillatory terms, one for each zero of the zeta function on the critical line and was derived by Riemann in his paper on primes assuming the Riemann hypothesis. We show that high resolution spectral lines can be generated by the truncated series at all powers of primes and demonstrate explicitly that the relative line intensities are correct. We then derive a Gaussian sum rule for Riemann's formula. This is used to analyze the numerical convergence of the truncated series. The connections to quantum chaos and semiclassical physics are discussed.

PACS numbers: 05.45.Mt, 02.10.De, 03.65.Sq, 02.70.Hm
\end{abstract}

Typeset by REVTEX 


\section{INTRODUCTION}

The Riemann zeta function is at the heart of number theory, and has also played a pivotal

role in the study of quantum chaos [1]. There is a deep connection between the complex zeros of the zeta function and Random Matrix Theory 2]. The zeros possess the same statistical properties as the energy eigenvalues of a dynamical Hamiltonian that is nonintegrable and whose dynamics are not time-reversal invariant. Unfortunately, this Hamiltonian is not known in terms of its dynamical variables. The main source of insight into this unknown quantum chaotic system comes from Gutzwiller's pioneering work [3], that expresses the oscillatory part of the quantum density of states as a sum over classical periodic orbits. (Such sums are now referred to as trace formulas.) It is well-known that the oscillatory part of the density of the Riemann zeros is given by a Gutzwiller-like sum, with one periodic term for every integer power of a prime number [4]. (A smoothed density of the Riemann zeros has also been studied in Ref. [5].) From this perspective, one can infer that a spectrum consisting of the Riemann zeros is generated by a Hamiltonian (albeit unknown) whose classical orbits have actions that are logarithms of primes and integer powers of primes.

Conversely, one could ask whether it is also possible to calculate the prime number sequence from a sum of oscillatory terms, with one term for every zero of the zeta function. Although less widely-known, such a series was actually given by Riemann himself [6]. Riemann derived an exact formula for the density of the primes (and their integer powers) that can be expressed as the sum of a smooth function and an infinite series of oscillatory terms involving the complex zeros of the zeta function. The smooth part has been thoroughly studied in the context of the prime number theorem whereas the oscillatory part has been largely ignored. Interestingly, it is the latter that contains the essential information about the location of the primes, as shown below. There is a vast literature on the distribution of the prime numbers. It is recognized that their distribution exhibits global regularity and local irregularity [7]. The nearest-neighbour spacings (NNS) of the primes is known to be Poisson-like [8], corresponding to an almost uncorrelated random distribution. This is very different in character from the Gaussian Unitary Ensemble (GUE) distribution of the Riemann zeros. Nevertheless, it is possible to generate the almost-uncorrelated sequence of all integer powers of primes from the interference of the highly-correlated Riemann zeros.

As mentioned above, from the perspective of semiclassical periodic orbit theory, the 
density of the Riemann zeros has the structure of a dynamical trace formula with periodic orbits. It is natural to ask whether Riemann's formula is a trace formula for the primes. Despite having the oscillatory terms, as discussed below, Riemann's formula is not a trace formula of dynamical origin. But, this does not preclude the existence of a trace formula for the primes. If one does exist, then this would support the notion that there exists a Hamiltonian system whose quantum spectrum is the primes. In any case, the exclusion of Riemann's formula as a trace formula suggests that there would be no correspondence between the classical dynamics and the Riemann zeros for this system.

The purpose of this paper is to study the density of the primes from the perspective of numerical semiclassics. To our knowledge, Riemann's series has not been studied numerically. We first verify that Riemann's formula does produce spectral lines at the positions of the primes and their powers, even when the series is truncated. This is not completely unexpected since Riemann's series converges conditionally to the exact density which is a set of $\delta$-function spikes. However, the $\delta$ functions arise from the entire series. The truncated series is an approximation to the exact density. It does not yield spikes, but rather lines of various widths, heights and (unknown) shapes and it is not at all obvious that the relative line intensities of the truncated series are correct. We examine this problem both numerically and analytically. We then provide a simple rule for estimating the value of the largest zero required to sufficiently resolve individual lines of a specific shape in some interval of interest and describe how to control the error incurred from a truncation of the series.

\section{RIEMANN'S FORMULA}

We start from the Euler product formula

$$
\zeta(\beta)=\prod_{p}\left(1-p^{-\beta}\right)^{-1}, \quad \operatorname{Re} \beta>1
$$

where the product is over all primes $p$. It follows that $\sum_{p} \sum_{n=1}^{\infty} \frac{1}{n} \exp (-n \beta \ln p)=\ln \zeta(\beta)$. Dividing both sides by $\beta$ and then taking the inverse Laplace transform of both sides with respect to $E$, we immediately obtain

$$
N(E)=\sum_{p} \sum_{n} \frac{1}{n} \Theta\left(E-\ln p^{n}\right)=\frac{1}{2 \pi i} \int_{a-i \infty}^{a+i \infty} \frac{\ln \zeta(\beta)}{\beta} e^{\beta E} \mathrm{~d} \beta,
$$


where $a>1$. Riemann evaluated the RHS of Eq. (2) to obtain $N(E)$. Upon differentiation with respect to $E$ and the subsequent substitution $x=e^{E}$, we obtain the density $\rho(x)$ of $p^{n} / n$ along the real axis $x$ as

$$
\rho(x)=\frac{1}{\ln x}-\frac{1}{x\left(x^{2}-1\right) \ln x}-2 \sum_{\alpha>0} \frac{\cos (\alpha \ln x)}{x^{1 / 2} \ln x},
$$

where $x>1$. This formula assumes the Riemann hypothesis, which states that the infinite number of complex zeros of the zeta function all lie on the critical line $\beta=(1 / 2 \pm i \alpha)$, where $\alpha$ is real and positive. Note that explicit use of the symmetry of the complex zeros has been made to reduce the summation to cosine functions. A generalized version of the Riemann formula, where the zeros may lie anywhere in the critical strip is given in Ref. [9]. We shall denote the sum over the oscillatory terms on the RHS of Eq. (3) as $\tilde{\rho}(x)$. Since Eq. (3) is exact, it is clear that the $\delta$-function spikes of $\rho(x)$ must be generated from the interference of the terms in $\tilde{\rho}(x)$. From our experience in periodic orbit quantization [10], we know that a coarse-grained version of the exact density of states can be reproduced even from a truncated periodic orbit sum. Therefore, in the following section, we focus on the numerical analysis of the truncated series.

Before presenting the results, however, we briefly review the pioneering numerical work of Riesel and Göhl [1]]. The LHS of Eq. (21) is a set of step functions, with unit steps at every prime $p$, one-half steps at $p^{2}$, one-third steps at $p^{3}$ and so on, and may be obtained by taking the contour integral of $\ln \zeta(\beta) / \beta$ on the RHS of the equation. Riemann [6] denotes this function by $f(x)$ and Edwards by $J(x)$ [12]. The number of primes less than $x$, denoted by $\pi(x)$, may be expressed in terms of $f(x)$ as

$$
\pi(x)=\sum_{n=1}^{\infty} \frac{\mu(n) f\left(x^{1 / n}\right)}{n},
$$

where $\mu(n)$ is the Möbius function [12]. The modulating effect of the oscillatory terms due to the first twenty nine pairs of the complex Riemann zeros was numerically examined by Riesel and Göhl [1] in 1970. This early work already showed the approximate formation of the first few steps at the prime numbers, and modulations for some larger primes. Note that the series (41) requires a knowledge of the Möbius function, and is much more complicated than Eq. (3). Riesel and Göhl actually replaced the sum over the Möbius functions by the Gram series involving factorials which are difficult to compute accurately for large integers. In this paper, we shall rather study formula (3) for $\rho(x)$ since it contains more information 
than the formula for the density of the primes (no powers) which can be obtained from the derivative of $\pi(x)$.

\section{NUMERICS}

Numerically, we can only evaluate a finite number of terms from Riemann's infinite series. Although it would seem by inspection that all terms of the series are equally important and that there is no optimal ordering of the terms, Riemann states that the series is conditionally convergent and that it must be summed in order of increasing size of $\alpha$. (For any series whose convergence is conditional, the order of summation must be specified since different orderings produce different results.) In this case, the "natural order" is the correct one. Riemann goes on to state that with this ordering the truncated series should give an approximation to the density of primes (and their powers), but that using a different ordering, the resulting finite series can approach arbitrary real values. We have verified this numerically and found that using finite sets of zeros chosen according to different rules yields incorrect results. Thus, for the numerical work that follows, we use the correct ordering.

\section{A. Line Intensities of the Truncated Series}

We first computed Eq. (3) using the first $10^{4}$ zeros and observed lines at the positions of the primes and their powers for $x<5000$. However, for $x>2000$, many lines cannot be fully resolved and the signal eventually dies out. This is due to truncation since only a small number of zeros have been included. (This will be discussed in more detail below.) Nevertheless, even this small number of zeros yields narrow lines at the lowest primes. In Fig. 1. we display the result for $x \in[1.5,100]$. Although one can clearly observe lines at the positions of the primes, the relative intensities cannot be determined by inspection since the lineshapes are not uniform (see Fig. (2). This is a common problem in spectral analysis, and is often resolved by imposing a more uniform lineshape through convolution of the signal with an appropriate smooth "response function" 13]. The response function is typically a peaked function that falls to zero in both directions from its maximum. Gaussian functions are positive-definite and decay rapidly. They are also convenient to use since their shape only depends on a single parameter (the variance) and therefore can be easily controlled. 


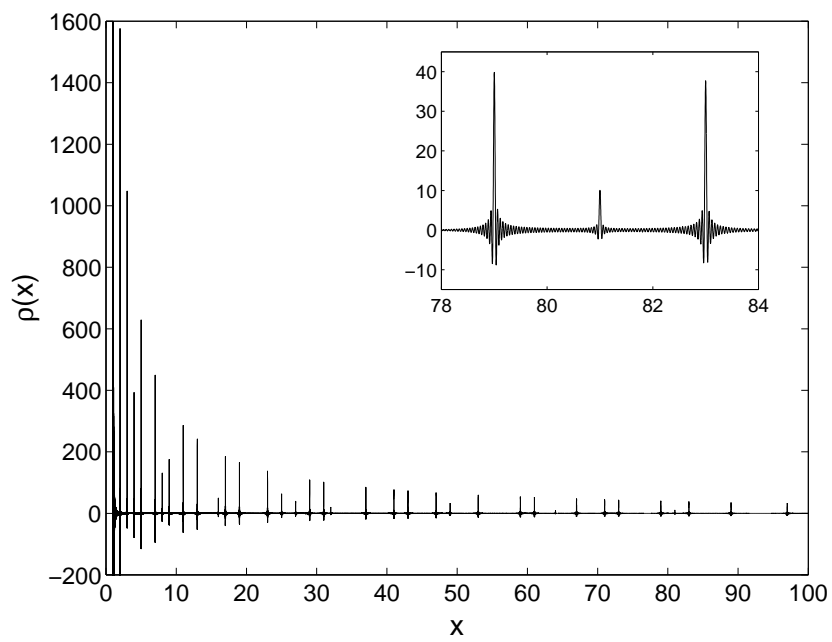

FIG. 1: The result of computing Eq. (3) using the first $10^{4}$ zeros for $x \in[1.5,100]$. The inset shows a closer view of three lines that appear for $x \in[77,84]$. The three lineshapes are similar so that their relative heights are somewhat meaningful. However, the lineshapes vary considerably throughout the entire range so that heights cannot be immediately interpreted as relative intensities.
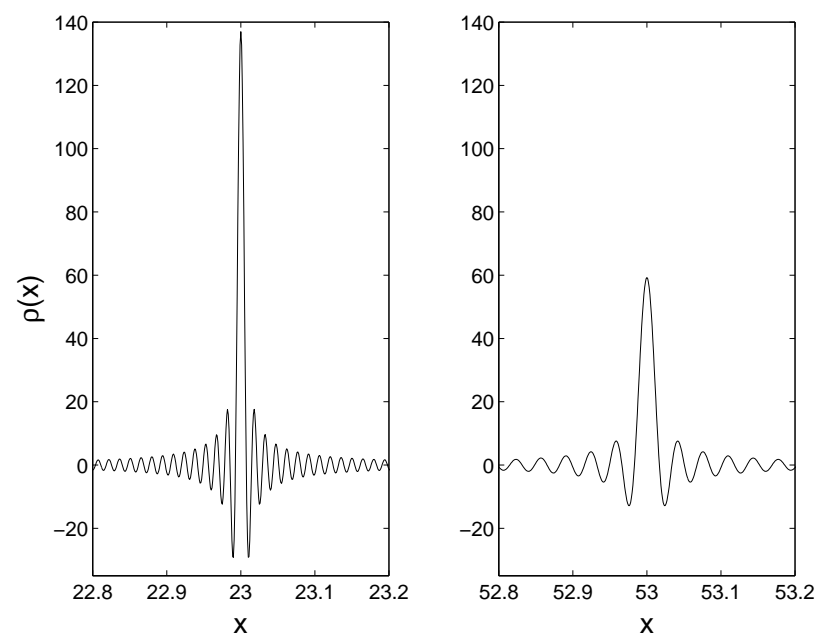

FIG. 2: A closer view of two non-adjacent lines in Fig. 1. The lineshapes clearly differ so that their relative heights are not meaningful.

Thus, we next convolve this approximate density $\rho(x)$ (i.e. smooth term and truncated series) with an unnormalized Gaussian of variance $\sigma$ :

$$
\rho(x) * G_{\sigma}(x)=\int_{1}^{\infty} \rho\left(x^{\prime}\right) G_{\sigma}\left(x-x^{\prime}\right) \mathrm{d} x^{\prime},
$$

where

$$
G_{\sigma}(x)=\exp \left(-x^{2} / 2 \sigma^{2}\right)
$$


The effect of the convolution is that rapidly oscillating features are washed out and smooth peaked features are smeared into the shape of the response function. If the lines were perfect $\delta$ functions of height $D_{n}$, then from Eq. (15), these would be replaced by $D_{n} G_{\sigma}\left(x-x_{n}\right)$, i.e. Gaussians of variance $\sigma$ with height $D_{n}$ at $x=x_{n}$. Of course, the lines are not $\delta$ function spikes so that the resultant lineshapes are not exactly Gaussian, but as long as the intrinsic linewidth is sufficiently small compared to the variance, the deviation from a perfect Gaussian is quite negligible. Therefore, the convolution produces a series of Gaussian lines, each of the same width. The key point is that the lineshapes are now essentially uniform so that the actual heights can be meaningfully compared and immediately interpreted as the relative intensities. It is important to keep in mind that since the response function has a maximum height of unity, the height of a line after convolution should be the area under that line before convolution. The reason for this is that although the lines of the original signal act like $\delta$ functions with respect to the response function, they do have nonzero widths and so their effective $\delta$-function "heights" $D_{n}$, are equal to the areas. In this sense, the convolution procedure is equivalent to directly integrating the area under each line of the signal. However, the convolution technique is much simpler and avoids errors that can arise from the long oscillatory tails of individual lines. Note that this procedure cannot resolve two adjacent lines when the spacing between them is smaller than $\sigma$ and thus $\sigma_{\max }=1 / 2$.

We have computed Eq. (5) for the range of interest in Fig. 1, This is shown in Fig. 3] using $\sigma=0.05$. We note here that the heights do not depend on the specific value of $\sigma$ due to the fact the Guassian is not normalized. As more terms are included in the sum, the natural linewidths decrease and the convolution becomes more accurate. It is then possible to produce high-resolution lines by using smaller variances. For example, using $10^{5}$ zeros, we produced lines with a variance $\sigma=0.01$. It would be useful to know how many primes can be resolved using a prescribed number of zeros. In the present scheme, one simply observes where the lines of the original signal develop a sufficiently large width. The important criterion here is that all linewidths should be at least smaller than the mean spacing between all powers of primes in the interval of interest. Of course, the width of any line is related to the number of terms used in truncating the series. Although this relationship can be determined, there is still the problem that all the lines have different shapes. Thus, we shall find it more useful to determine for what values of $x$ the truncated formula can no longer produce lines of a specific uniform shape. 

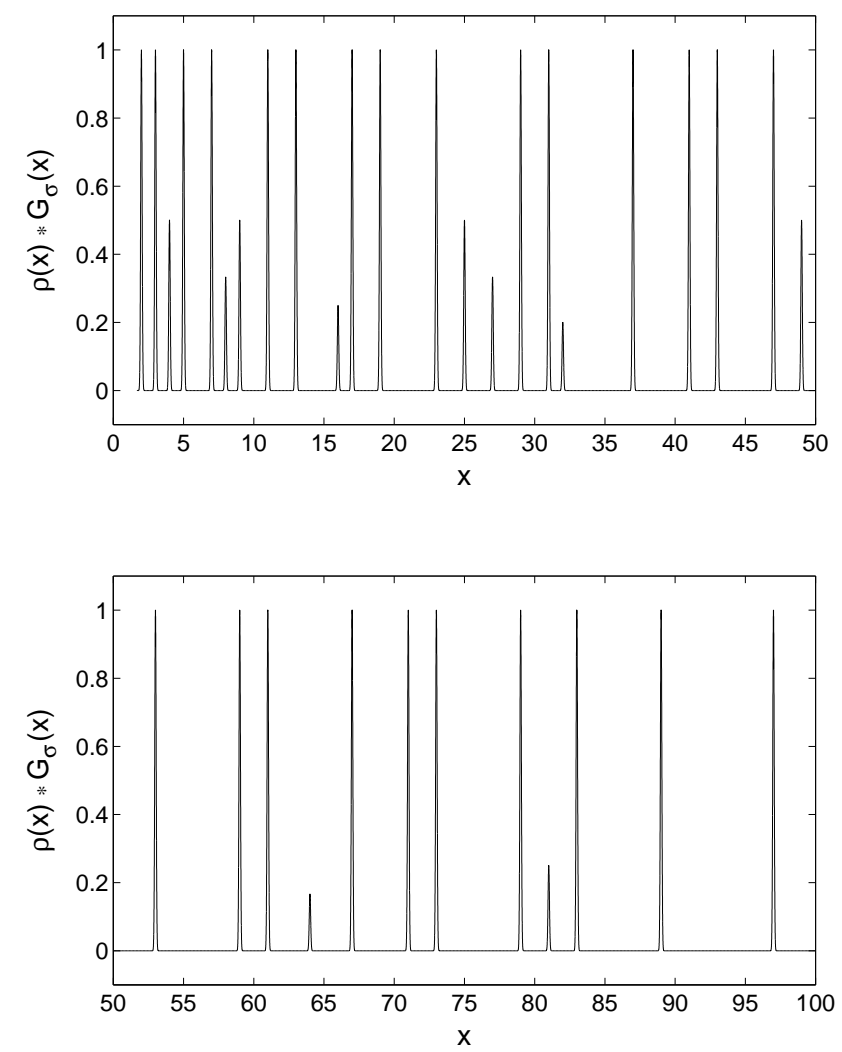

FIG. 3: The result of computing Eq. (15) using the first $10^{4}$ zeros and $\sigma=0.05$. The range is the same as in Fig. 1] Note that lines with height less than unity occur at powers of primes $p^{n}$ and have height $1 / n$ (for example, the line at $2^{6}=64$ has height $1 / 6=0.1 \dot{6}$ ).

\section{B. Sum Rule and Numerical Convergence}

The convergence of the series can be examined using a more controlled application of the convolution procedure described above. The general idea is to construct a series ("sum rule") that absolutely converges to a "coarse-grained" version of the exact density. This density is obtained by replacing all spikes of the exact density by smooth peaked functions. One immediate advantage is improved convergence since it is easier to reproduce the welldefined smooth peaked functions of a coarse-grained density using a truncated sum rule than it is to reproduce spikes using the original truncated series. However, for our purposes, the more important reason for using a sum rule is to control the convergence of the series. This will become evident after the sum rule is given. The sum rule itself is obtained from a direct convolution of the original series with a "smoothing function", that is, some smooth function whose Fourier coefficients rapidly decrease. (Since the original series consists of cosines, the 
resulting integral is essentially a cosine transform of the smoothing function.)

The above discussion is quite general. We now connect this idea with the numerical calculations described above. Assume the coarse-grained density consists of a set of Gaussian functions of variance $\sigma$ centered at each prime (or prime power) with heights equal to unity (or the reciprocal power). We want to construct a series as described above that converges to this density, that is, we want to find a Gaussian sum rule for Riemann's series. To do this, we convolve Riemann's series term-by-term with a Gaussian smoothing function. For the following calculation, we will define $S_{\alpha}(x)=\alpha \ln x$ and $A(x)=-2 / \sqrt{x} \ln x$. Then, we can write Riemann's formula as $\tilde{\rho}(x)=A(x) \sum_{\alpha} \operatorname{Re}\left\{\exp \left[i S_{\alpha}(x)\right]\right\}$. The Gaussian sum rule is

$$
\begin{aligned}
\tilde{\rho}_{\sigma}(x) & =\tilde{\rho}(x) * G_{\sigma}(x)=\int_{-\infty}^{\infty} \tilde{\rho}\left(x^{\prime}\right) e^{-\left(x-x^{\prime}\right)^{2} / 2 \sigma^{2}} \mathrm{~d} x^{\prime} \\
& =\sum_{\alpha} \operatorname{Re}\left\{\int_{-\infty}^{\infty} A\left(x^{\prime}\right) e^{i S_{\alpha}\left(x^{\prime}\right)} e^{-\left(x-x^{\prime}\right)^{2} / 2 \sigma^{2}} \mathrm{~d} x^{\prime}\right\}, \quad x>1 .
\end{aligned}
$$

For $\sigma \leq \sigma_{\max }=1 / 2$, the Gaussian rapidly decays to zero. This implies that the main contribution to the integral comes from a small interval centered about $x^{\prime}=x$. Elsewhere, the integrand is practically zero. Thus, we make two approximations to proceed further. First, the amplitude function $A\left(x^{\prime}\right)$ changes very slowly and on the small interval of interest $A\left(x^{\prime}\right) \approx A(x)$. Secondly, the phase function $S_{\alpha}\left(x^{\prime}\right)$ can be replaced by its Taylor series expansion about $x^{\prime}=x$ : $S_{\alpha}\left(x^{\prime}\right)=S_{\alpha}(x)+S_{\alpha}^{\prime}(x)\left(x^{\prime}-x\right)+\ldots$ If we retain the leading-order term only,

$$
\begin{aligned}
\tilde{\rho}_{\sigma}(x) & \approx A(x) \sum_{\alpha} \operatorname{Re}\left\{\int_{-\infty}^{\infty} e^{i\left[S_{\alpha}(x)+S_{\alpha}^{\prime}(x)\left(x^{\prime}-x\right)\right]} e^{-\left(x-x^{\prime}\right)^{2} / 2 \sigma^{2}} \mathrm{~d} x^{\prime}\right\} \\
& =A(x) \sum_{\alpha} \operatorname{Re}\left\{e^{i\left[S_{\alpha}(x)-x S_{\alpha}^{\prime}(x)\right]} e^{-x^{2} / 2 \sigma^{2}} \int_{-\infty}^{\infty} e^{-\left[x^{\prime 2}-\left(2 x+2 i \sigma^{2} S_{\alpha}^{\prime}(x)\right) x^{\prime}\right] / 2 \sigma^{2}} \mathrm{~d} x^{\prime}\right\} \\
& =\sqrt{2 \pi} \sigma A(x) \sum_{\alpha} e^{-\sigma^{2} S_{\alpha}^{\prime 2}(x) / 2} \operatorname{Re}\left\{e^{i S_{\alpha}(x)}\right\}
\end{aligned}
$$

where we have used the standard result for the Gaussian integral [14]. Finally, the Gaussian sum rule for Riemann's series is

$$
\tilde{\rho}_{\sigma}(x)=-\frac{2 \sqrt{2 \pi} \sigma}{\sqrt{x} \ln x} \sum_{\alpha} e^{-\sigma^{2} \alpha^{2} / 2 x^{2}} \cos (\alpha \ln x) .
$$

This sum rule explicitly shows the effect of convolution on the series; each term is modulated by an exponential factor. This factor essentially controls the convergence of the series for 
all values of $x$. Although the orginal series is only conditionally convergent, as long as the correct ordering is used, this sum rule is also absolutely convergent. As stated above, we seek an approximate relation between the maximum zero included in the sum and the maximum prime that can be resolved. One way to determine this is as follows. First, specify the value of the largest zero, $\alpha_{\max }$ and include all zeros $\alpha \leq \alpha_{\max }$. Then, there exists a set of values $x<x_{\max }$ for which the exponential factor falls below some threshold parameter $\varepsilon$. This condition immediately gives the simple relation

$$
x_{\max }=\left[\frac{\sigma}{\sqrt{-2 \ln (\varepsilon)}}\right] \alpha_{\max },
$$

where $0<\varepsilon<M$. For $\alpha>\alpha_{\max }$ and $x \leq x_{\max }$, all terms are exponentially smaller than $\varepsilon$ and are thus numerically insignificant. The choice of the parameter $\varepsilon$ depends on the desired precision of a resolved line. An upper bound $M$ for the parameter is the value of the exponential factor $\left(e^{-3 / 2}\right)$ at its inflection point $x_{I}=(1 / \sqrt{3}) \sigma \alpha[15]$. This implies $x_{\max }<x_{I}$. The lower bound can be as small as machine zero (for example $10^{-16}$ ). However, there is no reason for such an extreme choice since we are mostly interested in determining where numerical errors become significant (i.e. where lines are no longer visibly resolved and the intensities are erroneous by more than 1\%). Of course, higher precision can be imposed at the cost of resolving fewer primes. But, since the improved precision will not be apparent in the graph of $\rho_{\sigma}(x)$, there is no compelling reason to choose exceedingly small values. For our purposes, a convenient choice is $\varepsilon=e^{-7 / 2}$.

We now provide a few examples to illustrate the utility of relation (10). As a first example, we take $\alpha_{\text {max }} \doteq 9878$ which is the $10^{4}$ th zero. Using the above formula (with $\sigma=0.1$ ) yields $x_{\max } \doteq 373$. In Fig. 4, we evaluate Eq. (9) using the first $10^{4}$ zeros (and include the smooth term). One can clearly see significant errors for $x>400$. As a second example, we take $\alpha_{\max } \doteq 74921$ (the $10^{5}$ th zero). The formula then gives $x_{\max } \doteq 2832$. In Fig. [5] we truncate Eq. (9) at this value of $\alpha_{\max }$ and observe significant errors occur for $x>2900$.

An additional benefit of the sum rule is that it gives us an immediate measure of the error incurred from truncation. The largest errors are in the vicinity of $x_{\max }$ where there are contributions $O(\varepsilon)$ that have been excluded. For all other values of $x<x_{\max }$, the excluded terms are exponentially smaller. Of course, we have complete control of this error through our freedom in specifying $\varepsilon$. In the case of the original truncated series, it is not immediately 

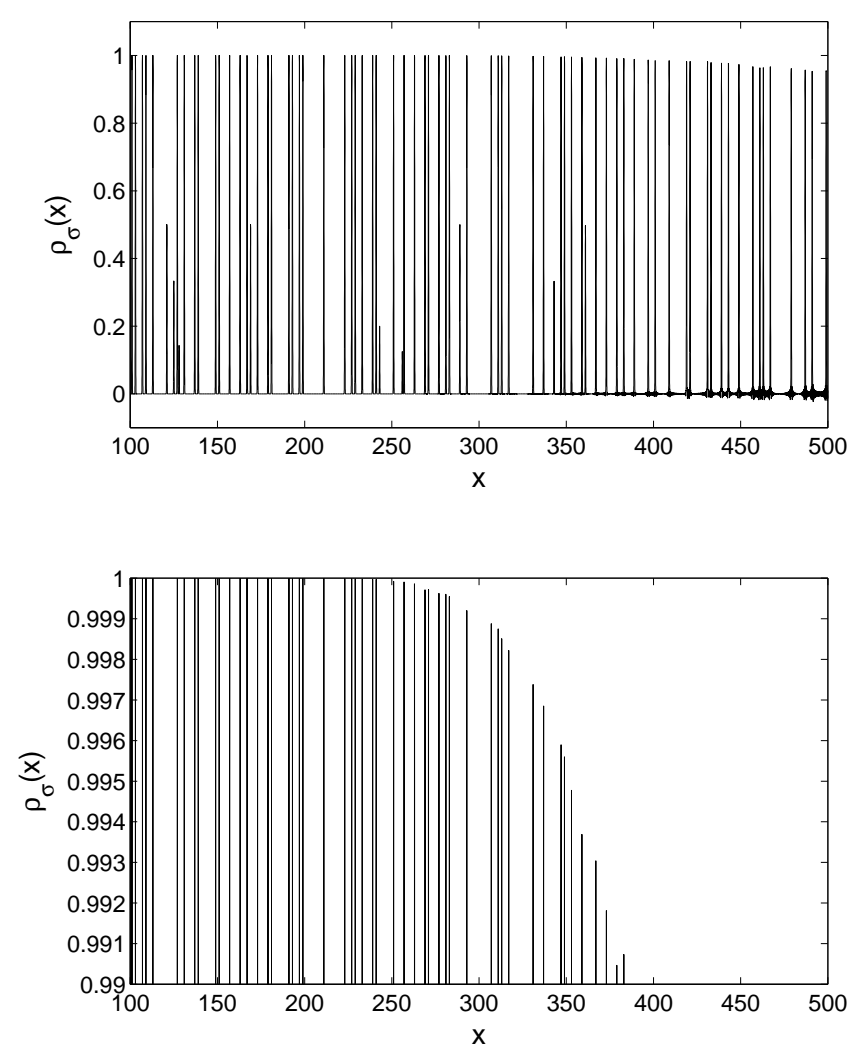

FIG. 4: The sum rule (9) using the first $10^{4}$ zeros and $\sigma=0.1$. The lower window shows a closer view of the lines in the interval $0.99<\rho_{\sigma}(x)<1$. Formula (10) indicates that for $x \leq x_{\max } \doteq 373$, all lines should be resolved and intensities should have errors less than $\sigma^{2}=0.01$.

obvious what the errors are, but they can be determined through more elaborate analysis.

\section{DISCUSSION AND CONCLUSION}

By writing $\zeta(\gamma+i t)=|\zeta(\gamma+i t)| \exp \left(-i \theta_{\gamma}(t)\right)$, we see that all the information about the zeros along the $t$-axis is contained in the phase $\theta_{\gamma}(t)$. This has to jump by $\pi$ to accomodate the sign change in $\zeta$ at every zero, and it can be shown that the oscillating part of the density of the zeros on the critical line is proportional to the derivative of the imaginry part of $\ln \zeta(t)$ with respect to $t[10]$. On the other hand, we see from Eq. (2) that the appropriate contour integral over $\ln \zeta(\beta)$ also yields $\rho(x)$ relating to the primes. Thus, the phase of the zeta function, as defined above, connects the Riemann zeros to the primes.

As mentioned above, if the series is truncated, the signal gradually dies out as $x$ increases. This can be understood by noting that due to the logarithmic dependence, each term pro- 

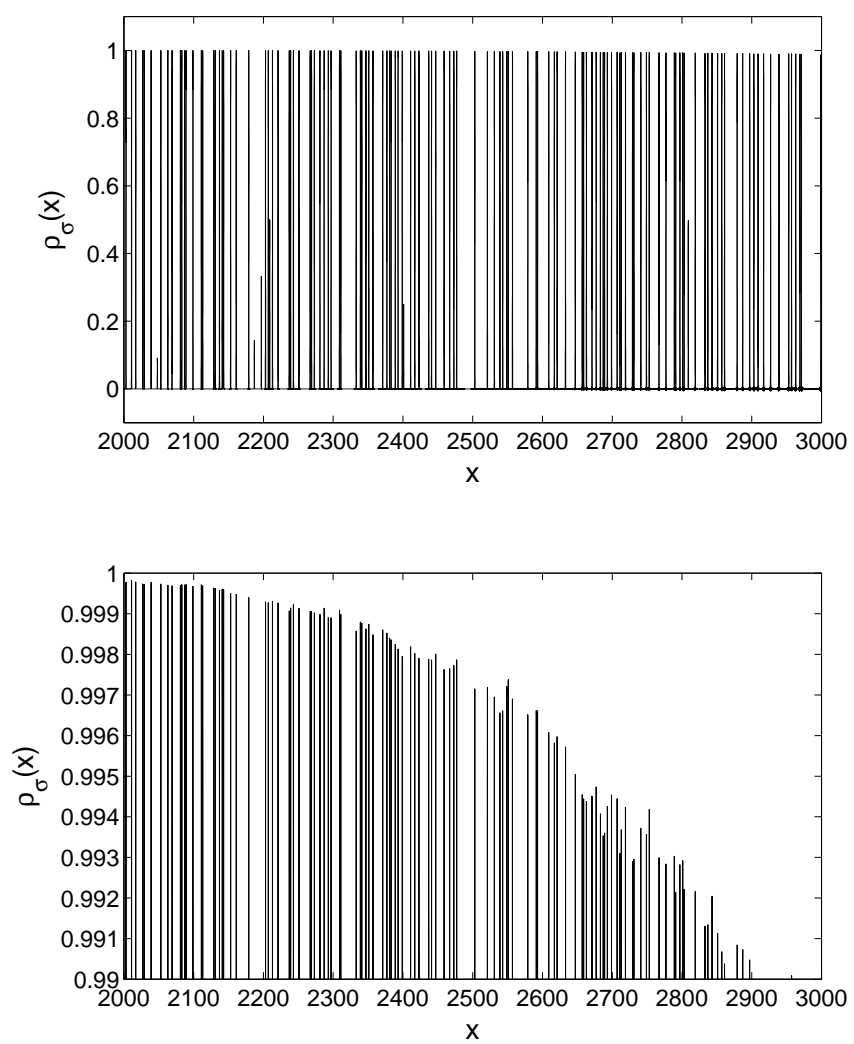

FIG. 5: The sum rule (9) using the first $10^{5}$ zeros and $\sigma=0.1$. The lower window shows a closer view of the lines in the interval $0.99<\rho_{\sigma}(x)<1$. Formula (10) indicates that for $x \leq x_{\max } \doteq 2832$, all lines should be resolved and intensities should have errors less than $\sigma^{2}=0.01$.

duces an oscillation whose period continually increases while its amplitude decays. Clearly, more high frequency (large $\alpha$ ) terms are required for sufficient constructive interference. This explains the fact that lines at small values of $x$ are resolved more quickly than at larger values. Although the higher frequency terms are responsible for short-range oscillations and one could imagine exclusive use of those terms rather than lower frequency terms, the difficulty is the conditional convergence of the series and the fact that all of the terms are equally important. Unfortunately, this implies that Riemann's formula is impractical for resolving lines at large primes. This is also consistent with Eq. (10). If one is interested in using Riemann's series to find (new) large primes, for example, on the order of 10 250,000 , then one requires an accurate knowledge of roughly the same number of zeros.

We emphasize that Riemann's formula, is only correct if the Riemann hypothesis is true. Otherwise, if a pair of zeros occur at $\beta_{ \pm}=\gamma \pm i \alpha$, the factor $x^{1 / 2}$ in the denominator of the oscillating term of Eq. (플 should be replaced by $x^{(1-\gamma)}[9]$. An interesting numerical 
experiment is to move the zeros off the critical line, that is, to arbitrarily change their real parts. We find this still produces lines at the primes and their powers, but the relative intensities are incorrect. This is interesting since it demonstrates that the location of the primes depends only on the imaginary part of the zero. The real part only affects the intensities which are anyway not evident from a direct evaluation of the series. This provides another motivation for the numerical and analytical procedures described in this paper.

It is natural to compare the oscillating part of the density $\tilde{\rho}(x)$ with the semiclassical trace formula [3, 16] of a dynamical system. One could identify $\alpha$ as an orbit label, one for each zero of the zeta function and $x$ as the single-particle energy variable. Then, $\rho(x)$ in Eq. (3) may be interpreted as the density of states as a function of energy with the first term on the RHS corresponding to the smooth Thomas-Fermi (TF) contribution [17]. In the oscillating part, the argument $\alpha \ln x$ of the cosine term should then correspond to the action $S_{\alpha}(x)$ of the orbit $\alpha$. Note however that there are no implicit repetition indices in Eq. (3) thereby implying that even if one gives a dynamical interpretation to $\tilde{\rho}(x)$, the orbits are not periodic. This is in direct contrast to the trace formula for the Riemann zeros, in which the orbits are periodic with primitive period $\ln p$ for each prime [4]. Of course, the most striking feature is that the amplitude has no $\alpha$ dependence. Even oscillatory contributions to the density of states from non-periodic trajectories usually have amplitudes that depend on the orbit [18]. In the event that there is a fortuitous cancellation of the index $\alpha$, it is unlikely that the energy dependence in the denominator of the oscillating term as well as the TF term can then be generated consistently by the same Hamiltonian. Consequently, Riemann's formula is not a trace formula of dynamical origin.

With regard to spectral statistics, it is well known that nearest-neighbour spacings (NNS) 19] of the Riemann zeros obey the GUE distribution of Random Matrix Theory, characteristic of a chaotic quantum system without time-reversal symmetry [20, 21]. The same zeros also generate the spectrum of the primes and their powers through Riemann's formula (3). As mentioned earlier, the NNS distribution of the primes is Poisson-like [8], with some level repulsion, which, if at all of dynamic origin, hints only to near-integrability 1]. Thus, it is quite remarkable that the highly-correlated sequence of the zeros can (through Riemann's formula) interfere to produce the almost-uncorrelated sequence of the primes.

In conclusion, we have demonstrated that the spectrum of the primes and their integer powers can be accurately generated from a sum of periodic terms, each term involving a 
zero of the zeta function. This is in the spirit of semiclassical periodic orbit theory, where the individual levels of a quantum spectrum may be resolved from a sum of oscillatory terms, each arising from periodic orbits. Despite the accuracy of the generated spectrum, Riemann's formula is not a trace formula. However, this does not imply that there is no such formula, and it would still be interesting to understand the spectrum of the primes in terms of periodic orbits. This could provide insight into the structure of a possible trace formula for the primes. If this formula could be found, the remaining challenge would be to obtain the corresponding Hamiltonian.

\section{Acknowledgments}

We acknowledge Ranjan Bhaduri, Randy Dumont, Avinash Khare, John Nieminen, Muoi Tran, Jim Waddington for useful discussions, and Andrew Odlyzko for supplying the Riemann zeros. This work was financially supported by the Natural Sciences and Engineering Research Council of Canada (NSERC).

[1] H.-J. Stöckmann Quantum Chaos, (Cambridge University Press, Cambridge, 1999).

[2] J. P. Keating and N. C. Snaith, Commun. Math. Phys. 214, 57 (2000); P. Diaconis, Bull. Am. Math. Soc. 40, 155 (2003).

[3] M. C. Gutzwiller, J. Math. Phys. 12, 343 (1971).

[4] M. V. Berry, in Quantum Chaos and Statistical Nuclear Physics, T. H. Seligman and H. Nishioka (eds.), Lecture Notes in Physics 263 (Springer-Verlag, Berlin, 1986).

[5] R. K. Bhaduri, A. Khare, S. M. Reimann, and E. L. Tomusiak, Ann. Phys. (N.Y.) 254, 25 (1997).

[6] B. Riemann, Monatsber. Akad. Berlin, 671-680 (1859); the English translation can be found in the appendix of Ref. [12].

[7] G. Tenebaum and M. M. France, The Prime Numbers and Their Distributions (American Mathematical Society, 2000).

[8] C. E. Porter, Statistical Theories of Spectra : Fluctuations (Academic Press, New York, 1965).

[9] M. V. Berry and J. P. Keating, SIAM Rev. 41, 236 (1999). 
[10] M. Brack and R. K. Bhaduri, Semiclassical Physics (Addison-Wesley, Reading, Mass., 1997).

[11] H. Riesel and G. Göhl, Math. Comp. 24, 969 (1971).

[12] H. M. Edwards, Riemann's Zeta Function (Academic Press, New York, 1974); Dover Reprint, 2001.

[13] H. J. Nussbaumer, Fast Fourier Transform and Convolution Algorithms (Springer-Verlag, New York, 1982).

[14] $\int_{-\infty}^{\infty} e^{-a x^{\prime 2}+b x^{\prime}} \mathrm{d} x^{\prime}=\sqrt{\frac{\pi}{a}} e^{b^{2} / 4 a} \quad(a>0)$. It is not necessary to evaluate the convolution integral over the interval $(1, \infty)$. If one insisted on doing so, the integral at the end is rather $\int_{1}^{\infty} e^{-a x^{\prime 2}+b x^{\prime}} \mathrm{d} x^{\prime}=\sqrt{\frac{\pi}{4 a}} e^{b^{2}} / 4 a \operatorname{erfc}\left(-\frac{b}{2 \sqrt{a}}+\sqrt{a}\right)$. The resulting sum rule is slightly more complicated, but the difference is insignificant since $\sigma$ is small.

[15] For smaller values of $x$, a more accurate upper bound is given by the inflection point of $x^{-1 / 2} e^{-\sigma^{2} \alpha^{2} / 2 x^{2}}$ which is $\frac{\sqrt{2(12-3 \sqrt{13})}}{3} \sigma \alpha$. If the logarithmic term is also included, the inflection points are roots of a transcendental equation.

[16] M. C. Gutzwiller, Chaos in Classical and Quantum Mechanics (Springer-Verlag, New York, 1990).

[17] The second smooth term on the RHS of Eq. (31) arises from the trivial zeros, and may not be taken as part of a TF term.

[18] R. Aurich, T. Hesse, and F. Steiner, Phys. Rev. Lett. 74, 22 (1995); W. Zheng, Phys. Rev. E 60, 2845 (1999); D. Biswas, Phys. Rev. E 61, 5129 (2000); D. Biswas, Phys. Rev. E 63, $016213(2001)$.

[19] O. Bohigas, M. J. Giannoni, and C. Schmit, Phys. Rev. Lett. 52, 1 (1984).

[20] A. M. Odlyzko, Math. Comput. 48, 273 (1987).

[21] M. L. Mehta, Random Matrices (Academic Press, New York, 1991). 